\title{
Single transverse-spin asymmetries in forward pion production at high energy: incorporating small- $x$ effects in the target
}

\author{
Daniël Boel* \\ Dept. of Physics and Astronomy, Vrije Universiteit Amsterdam, \\ De Boelelaan 1081, $1081 \mathrm{HV}$ Amsterdam, The Netherlands \\ Adrian Dumitruf and Arata Hayashigak \\ Institut für Theoretische Physik, J. W. Goethe Universität \\ Max-von-Laue Strasse 1, D-60438 Frankfurt am Main, Germany
}

(Dated: April 21, 2018)

\begin{abstract}
We consider single-inclusive forward pion production in high-energy proton-proton collisions at RHIC energies. A good baseline description of the transverse momentum distributions at high rapidity is obtained within Mueller's dipole formalism with an anomalous dimension incorporating an "extended geometric scaling" window between the saturation and DGLAP regimes. We then formulate pion production for transversely polarized projectiles within the same approach. We assume that an azimuthal, spin-dependent asymmetry arises from the so-called Sivers effect and investigate the single transverse-spin asymmetry $A_{N}$ at $200 \mathrm{GeV}$ and $500 \mathrm{GeV}$ center-of-mass energy. A simple parameterization of the Sivers functions from the literature compares reasonably well with the high-energy STAR data if the overall normalization is scaled up by at least a factor of two. The STAR data might therefore indicate that the Sivers effect is significantly stronger than thought so far. We also analyze higher-twist contributions to $A_{N}$ and find that they largely cancel.
\end{abstract}

PACS numbers: 12.38.-t,12.38.Bx,13.88.+e,13.85.-t

\section{INTRODUCTION}

Large single transverse-spin asymmetries (SSAs) in $p^{\uparrow} p \rightarrow \pi X$ have been observed in fixed target experiments [1] over the last 15 years. A non-vanishing SSA implies that the azimuthal distribution of pions in the final state depends on the direction of the transverse spin of the polarized projectile proton. These observations pose a challenge for theorists who want to explain such asymmetries in terms of quark and gluon degrees of freedom. It is clear that the asymmetries result from spin-orbit couplings and chiral dynamics in low-energy QCD, but to describe such couplings and chiral symmetry breaking within quantum field theory is far from straightforward. Attempts to describe the observable

$$
A_{N}=\frac{\mathrm{d} \sigma\left(p^{\uparrow} p \rightarrow \pi X\right)-\mathrm{d} \sigma\left(p^{\downarrow} p \rightarrow \pi X\right)}{\mathrm{d} \sigma\left(p^{\uparrow} p \rightarrow \pi X\right)+\mathrm{d} \sigma\left(p^{\downarrow} p \rightarrow \pi X\right)}
$$

perturbatively at leading twist and within the framework of collinear factorization where partonic transverse momenta in hadrons are assumed to play a subdominant role (because the associated scale is of order $\Lambda_{Q C D}$ ), result in very small asymmetries [2]. Several proposals that go beyond leading twist or collinear factorization have been put forward [3, 4, 5, 6] and can in principle account for the large asymmetries. They all involve new polarization-dependent parton distribution or fragmentation functions, which however cannot be calculated within perturbative QCD (pQCD) because of the soft dynamics. So far, these functions could also not be calculated from Euclidean QCD on a lattice. Hence, reliable predictions can only be made once the unknown functions have been extracted from experiments. As a further complication the evolution properties of the functions, needed to

\footnotetext{
*Electronic address: D.Boer@few.vu.nl

${ }^{\dagger}$ Electronic address: dumitru@th.physik.uni-frankfurt.de

‡Electronic address: aratah@th.physik.uni-frankfurt.de
} 
describe the energy dependence of the asymmetry observables, are also not known yet, because of the much more complicated operator structure as compared to the ordinary parton densities. Despite these limitations, a fairly successful phenomenology has emerged. Given the large magnitude of the effects it is certainly worth exploring such SSAs further, both experimentally and theoretically.

Due to the rather low energies and transverse momenta probed by fixed target experiments, a factorized pQCD description of the process may be somewhat questionable. Therefore, the high-energy collider experiments with polarized protons at BNL's Relativistic Heavy-Ion Collider (RHIC) were eagerly awaited. Recently, the STAR collaboration at RHIC has found that the large single spin asymmetries persist at collider energies of $\sqrt{s}=200 \mathrm{GeV}$ and transverse momenta up to $p_{T} \sim 2.5 \mathrm{GeV} / \mathrm{c}$ [7]. This has been confirmed by the BRAHMS experiment at RHIC 8]. Ref. 9] suggested that the asymmetries at low and high center of mass energies are actually two different phenomena, which was based on the observation that only at RHIC energies the unpolarized inclusive pion production cross section is described satisfactorily by a leading-twist, next-to-leading order (NLO) pQCD calculation. The fact that this does not work for fixed-target energies casts some doubt on the validity of the factorization assumption for those cases.

In this paper we shall specifically address the high-energy collider data on SSAs. As a first step, we attempt to obtain a good baseline for inclusive pion production in unpolarized $p p$ collisions. SSAs are usually measured at forward rapidities $y$ (in the hemisphere of the polarized projectile) where they are found to be largest. The large rapidity coverage of the forward $\pi^{0}$ detectors at STAR enable measurements of pion production up to $y \sim 4$ 10. At large $y$, however, the kinematics is very asymmetric: the hadrons in the final state emerge from collisions of projectile partons with large light-cone momentum fraction $x_{1} \sim 0.1$ - 1 with target partons carrying a very small momentum fraction $x_{2} \ll 1$. When $\alpha_{s} \log 1 / x_{2}$ is of order 1 , small- $x$ effects in the target become important and modify its gluon distribution function as compared to that obtained in the DGLAP approximation (specifically, its anomalous dimension). The purpose of this paper is to show how such small- $x$ effects can be accounted for in the description of SSAs at forward rapidities and high energies. Their relevance for deep inelastic scattering (DIS) at HERA and forward pion production in $p A$ collisions at RHIC has already been discussed in (among others) refs. [1, 12, 13] and [14, 15, 16, 17], respectively.

We will assume that the asymmetry arises predominantly from the so-called Sivers effect [3]. Within the framework of spin and transverse momentum dependent distribution and fragmentation functions, it has been shown [18] that other possible mechanisms, such as the Collins effect [4], cannot describe the asymmetries without other additional contributions, most importantly the Sivers effect. We therefore simply restrict to the Sivers effect, but inclusion of the other types of spin effects will proceed along similar lines.

The first study of small- $x$ effects, more specifically of saturation effects within the so-called "Color Glass Condensate" (CGC) formalism, on a single spin asymmetry concerned production of polarized $\Lambda$ baryons in collisions of unpolarized protons on nuclei 19]. No effects from small- $x$ quantum evolution, such as geometric scaling violations (see below), were taken into account though. Also, we shall show here that higher-twist saturation effects play no significant role for $p p$ collisions at RHIC energy, as may be expected from estimates of the saturation scale $Q_{s}$

This paper is organized as follows. In the next section we describe a model for the Sivers effect using a well-known parameterization taken from the literature. In sec. III-A we formulate single-inclusive forward hadron production in unpolarized $p p$ collisions within the CGC formalism and show that good agreement with the experimental data emerges. Next, by applying the formalism to $p^{\uparrow} p$ collisions, in sec. III-B we compare our results for the SSA to recent STAR data at large rapidity $(\simeq 3.8)$. Sec. IV is devoted to a discussion of the importance of higher-twist effects and finally a summary is given in sec. $\mathrm{V}$.

\section{A MODEL FOR THE SIVERS EFFECT}

The Sivers effect is a correlation between the direction of the transverse spin of the proton and the transverse momenta of its unpolarized partons. The Sivers effect in the process $p^{\uparrow} p \rightarrow \pi X$ has first been analyzed by Anselmino et al. 5]. The Sivers functions for valence $u$ and $d$ quarks were extracted from a fit to fixed-target data under the assumption that the asymmetry arises solely from this effect. That fit relied on a calculation of both the polarized and unpolarized cross sections within the LO DGLAP pQCD approach and partial inclusion of partonic transverse momentum dependence. In the present paper we will analyze whether the Sivers function 
from [5] is consistent with the high-energy STAR data, when incorporated in the CGC formalism. To be precise, we shall use the Sivers functions presented in refs. [20, 21]. Although updated fits 22] incorporate more detailed transverse momentum dependence, the simpler parameterizations of [20, 21] will suffice for our present purposes. The number densities of $q=u, \bar{u}, d, \bar{d}, \ldots$ partons in a polarized proton are defined as 22]

$$
f_{q / p^{\uparrow}}\left(x, \vec{k}_{t}\right)=f_{q / p}\left(x, k_{t}\right)+\frac{1}{2} \Delta^{N} f_{q / p^{\uparrow}}\left(x, k_{t}\right) \frac{\vec{S}_{t} \cdot\left(\vec{P} \times \vec{k}_{t}\right)}{\left|\vec{S}_{t}\right||\vec{P}|\left|\vec{k}_{t}\right|},
$$

where $\vec{S}_{t}$ and $\vec{P}$ are the transverse polarization and three-momentum vectors of the proton, respectively, and $\vec{k}_{t}$ is the quark's intrinsic transverse momentum. The function $f_{q / p}\left(x, k_{t}\right)$ is the density of quarks with transverse momentum $k_{t}$ in an unpolarized proton, from which the familiar quark distribution $f_{q / p}(x)$ arises by integration over $k_{t}$, see below. $\Delta^{N} f_{q / p^{\uparrow}}\left(x, k_{t}\right)$ denotes the Sivers function (see ref. 23] for a comparison to another frequently used notation $f_{1 T}^{\perp}[24]$ ).

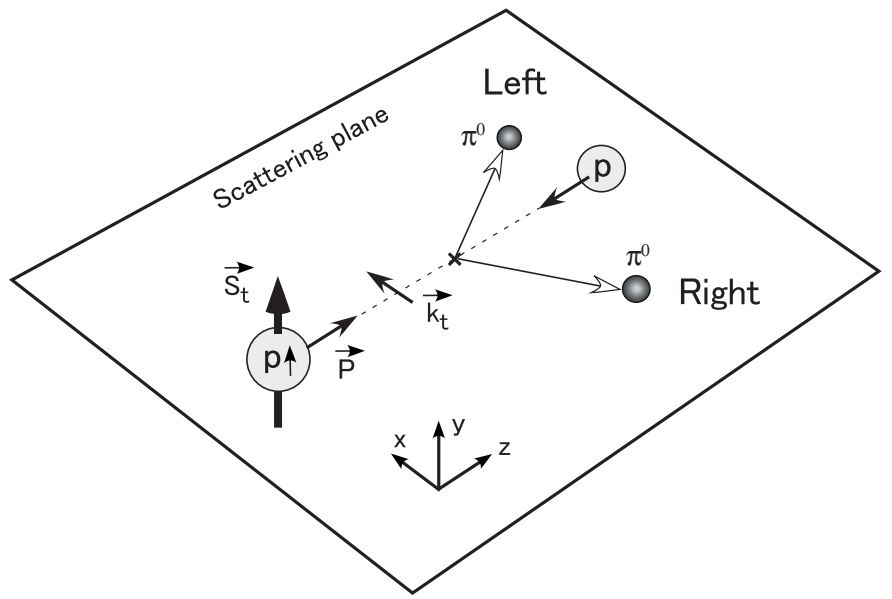

FIG. 1: Illustration of the $p^{\uparrow} p \rightarrow \pi^{0} X$ process. The kinematics is simplified by selecting the pion production plane $(x-z)$ perpendicular to the polarization $\vec{S}_{t}=\left(0, S_{y}, 0\right)$ of the proton. Parton transverse momenta are taken to be (anti-) parallel to the $x$-direction. We define left (right)-hand production of pions in the direction of $\vec{S}_{t} \times \vec{P}>0(<0)$ to match the experimental situation.

Fig. 11 illustrates the $p^{\uparrow} p \rightarrow \pi^{0} X$ collision process, simplifying the kinematics by taking the $\pi^{0}$ production plane $(x-z)$ perpendicular to the polarization of the proton, $\vec{S}_{t}=\left(0, S_{y}, 0\right)$, where the Sivers effect is maximal. The collision axis is along the $z$-direction, i.e. $\vec{P}=\left(0,0, P_{z}\right)$. Following the standard experimental definition, we define the left and right directions of $\pi^{0}$ production in the forward (positive hadron rapidity $y_{h}$ ) region from the perspective of the polarized proton as indicated in the figure. This definition implies that if $\Delta^{N} f_{q / p^{\uparrow}}\left(x, k_{t}\right)>0$, then the quark in the polarized proton has a preference for moving to the left, i.e. with $\vec{k}_{t}$ pointing in the positive $x$-direction.

The difference between upward and downward spin polarizations of the proton, which enters in the numerator of $A_{N}$, therefore picks up a leading contribution from the Sivers functions. Flipping the spin of the proton is equivalent to changing the sign of $\vec{k}_{t}$ :

$$
\begin{aligned}
\Delta^{N} f_{q / p^{\uparrow}}\left(x, \vec{k}_{t}\right) & \equiv f_{q / p^{\uparrow}}\left(x, \vec{k}_{t}\right)-f_{q / p^{\downarrow}}\left(x, \vec{k}_{t}\right)=f_{q / p^{\uparrow}}\left(x, \vec{k}_{t}\right)-f_{q / p^{\uparrow}}\left(x,-\vec{k}_{t}\right) \\
& =\Delta^{N} f_{q / p^{\uparrow}}\left(x, k_{t}\right) \frac{\vec{S}_{t} \cdot\left(\vec{P} \times \vec{k}_{t}\right)}{\left|\vec{S}_{t}\right||\vec{P}|\left|\vec{k}_{t}\right|},
\end{aligned}
$$

where $\Delta^{N} f_{q / p^{\uparrow}}\left(x, \vec{k}_{t}\right)$ is an odd function of $\vec{k}_{t}$. The Sivers effects cancel in the sum over polarization states, which 
enters in the denominator of $A_{N}$ and yields (twice) the leading-twist unpolarized function,

$$
f_{q / p^{\uparrow}}\left(x, \vec{k}_{t}\right)+f_{q / p^{\downarrow}}\left(x, \vec{k}_{t}\right)=2 f_{q / p}\left(x, k_{t}\right) .
$$

Note that $\int d^{2} k_{t} f_{q / p}\left(x, k_{t}\right)=f_{q / p}(x)$.

The inclusion of intrinsic $\vec{k}_{t}$-dependence in the parton distributions is not straightforward from a theoretical point of view. First of all, factorization theorems involving transverse momentum dependence are generally harder to derive. Considerable progress has been made for specific processes 25] which are, however, less complicated than the one considered here. We shall view the factorized formulas employed below as reasonable phenomenological extensions of collinear factorization at the semi-hard observed transverse momenta we are considering. Furthermore, we refer to ref. [16] for a derivation of collinear factorization from a one-loop analysis of unpolarized parton scattering off a CGC target.

Another important theoretical issue regarding Sivers functions is the calculable process dependence [26]. Since the fitted functions from refs. 20, 21] apply to the same process as considered here (and to the same partonic subprocess in the sense that $q g$ scattering is dominant in the considered kinematic region), we will not worry about this issue here.

As already mentioned above, the Sivers functions appear in the difference between upward and downward spin polarizations of the proton

$$
\begin{aligned}
d \sigma\left(p^{\uparrow} p \rightarrow h X\right)-d \sigma\left(p^{\downarrow} p \rightarrow h X\right) & \propto \int d^{2} k_{t}\left[f_{q / p^{\uparrow}}\left(x, \vec{k}_{t}\right)-f_{q / p \downarrow}\left(x, \vec{k}_{t}\right)\right] \otimes d \sigma^{q p \rightarrow q^{\prime} X}\left(\vec{k}_{t}\right) \otimes D_{h / q^{\prime}}\left(z_{h}\right) \\
& =\int d^{2} k_{t} \Delta^{N} f_{q / p^{\uparrow}}\left(x, \vec{k}_{t}\right) \otimes d \sigma^{q p \rightarrow q^{\prime} X}\left(\vec{k}_{t}\right) \otimes D_{h / q^{\prime}}\left(z_{h}\right)
\end{aligned}
$$

where $d \sigma^{q p \rightarrow q^{\prime} X}\left(\vec{k}_{t}\right)$ denotes the cross section of a parton scattering off an unpolarized proton. The fragmentation functions $D_{h / q^{\prime}}\left(z_{h}\right)$ describe the fragmentation of a final-state parton to a hadron with momentum fraction $z_{h}$. Note that the Sivers functions correspond to distributions of unpolarized partons and so the elementary hard-scattering cross sections are the usual expressions for unpolarized partons, in both the numerator and the denominator of $A_{N}$.

Because the convolution integrand has to be an even function of $\vec{k}_{t}$, while the Sivers function is odd, one finds

$$
\begin{aligned}
\int d^{2} k_{t} \Delta^{N} f_{q / p^{\uparrow}}\left(x, \vec{k}_{t}\right) \otimes d \sigma^{q p \rightarrow q^{\prime} X}\left(\vec{k}_{t}\right) \\
=\left(\int_{\hat{\vec{x}} \cdot \vec{k}_{t}>0}+\int_{\hat{\vec{x}} \cdot \vec{k}_{t}<0}\right) d^{2} k_{t} \Delta^{N} f_{q / p^{\uparrow}}\left(x, \vec{k}_{t}\right) \otimes d \sigma^{q p \rightarrow q^{\prime} X}\left(\vec{k}_{t}\right) \\
=\int_{\text {h.p. }} d^{2} k_{t} \Delta^{N} f_{q / p^{\uparrow}}\left(x, \vec{k}_{t}\right) \otimes\left[d \sigma^{q p \rightarrow q^{\prime} X}\left(\vec{k}_{t}\right)-d \sigma^{q p \rightarrow q^{\prime} X}\left(-\vec{k}_{t}\right)\right],
\end{aligned}
$$

where "h.p." indicates that the integration extends over only the positive half plane with $\hat{\vec{x}} \cdot \vec{k}_{t}>0$.

Following [5, 20] we assume that the Sivers function $\Delta^{N} f_{q / p^{\uparrow}}\left(x, \vec{k}_{t}\right)$ is sharply peaked about an average transverse momentum $k_{t}^{0}=\sqrt{\left\langle\vec{k}_{t}^{2}\right\rangle}$ such that

$$
\Delta^{N} f_{q / p^{\uparrow}}\left(x, \vec{k}_{t}\right) \simeq \Delta^{N} f_{q / p^{\uparrow}}(x) \delta^{(2)}\left(\vec{k}_{t}-k_{t}^{0} \hat{\vec{k}}_{t}\right)
$$

The unit vector $\hat{\vec{k}}_{t}$ is taken to lie in the positive half of the scattering plane, $\hat{\vec{x}} \cdot \hat{\vec{k}}_{t}>0$. Then

$$
\begin{aligned}
& \int d^{2} k_{t} \Delta^{N} f_{q / p^{\uparrow}}\left(x, \vec{k}_{t}\right) \otimes d \sigma^{q p \rightarrow q^{\prime} X}\left(\vec{k}_{t}\right) \otimes D_{h / q^{\prime}}\left(z_{h}\right) \\
& \simeq \Delta^{N} f_{q / p^{\uparrow}}(x) \otimes\left[d \sigma^{q p \rightarrow q^{\prime} X}\left(k_{t}^{0} \hat{k}_{t}\right)-d \sigma^{q p \rightarrow q^{\prime} X}\left(-k_{t}^{0} \hat{k}_{t}\right)\right] \otimes D_{h / q^{\prime}}\left(z_{h}\right) .
\end{aligned}
$$

Before the STAR data was published, the only available quantitative knowledge of the Sivers functions $\Delta^{N} f_{q / p^{\uparrow}}(x)$ was due to fits to SSA data from fixed-target $p p$ experiments and to low energy semi-inclusive 
DIS data, cf. ref. [27] and references therein. Although we will briefly comment on some more recent fits below, for this paper we will mainly restrict ourselves to the simpler parameterizations that resulted from fixed-target $p p$ data. We only allow for a different overall normalization of the Sivers functions. For valence quarks, the functions were parameterized as follows $[20,28]^{1}$ :

$$
\Delta^{N} f_{q / p^{\uparrow}}(x)=K_{\Delta f} \frac{k_{t}^{0}(x)}{M_{p}} N_{q} x^{a_{q}}(1-x)^{b_{q}} .
$$

The $x$-dependent average transverse momentum $k_{t}^{0}$ of valence quarks in the polarized proton is taken as

$$
k_{t}^{0}(x)=0.47 x^{0.68}(1-x)^{0.48} M_{p},
$$

for all flavors. $M_{p}=1 \mathrm{GeV}$ denotes the proton mass. The maximal intrinsic transverse momentum of $\approx 210 \mathrm{MeV}$ is on the order of the inverse proton radius and therefore reasonable. At large $x$, one does not expect much higher intrinsic transverse momenta. The remaining parameters are given by

$$
N_{u, d} / 4=(3.68,-1.24), \quad a_{u, d}=(1.34,0.76), \quad b_{u, d}=(3.58,4.14)
$$

It was pointed out in ref. 21] that the original values of $N_{q}$ from 20] were misquoted and have to be multiplied by a factor of 4 . In addition, in (9) we allow for further adjustment of the magnitude of the Sivers functions by a factor $K_{\Delta f}$ if required by the new high-energy data from STAR.

\section{FORWARD PARTICLE PRODUCTION AT HIGH ENERGIES}

\section{A. Unpolarized $p p$ collisions}

The forward pion spectra in $p p$ collisions obtained by the STAR Collaboration at RHIC $(\sqrt{s}=200 \mathrm{GeV})[29]$ are generally known to agree reasonably well with leading-twist NLO pQCD calculations in the DGLAP approximation 9, 30]. Although this approach is recognized as providing a much better description of semi-hard particle production at RHIC than at lower energy [9], not all commonly employed sets of parton distribution and fragmentation functions (FFs) perform equally well. To be specific, the NLO pQCD result with CTEQ6M PDFs and KKP FFs is consistent with the data for rapidity $\langle\eta\rangle=3.8$, but compares less well at $\langle\eta\rangle=4.0$, except in the high- $p_{t}$ region. The Kretzer FFs lead to better agreement with lower- $p_{t}$ data, presumably due to its smaller gluon fragmentation to pions as compared to the KKP set, which may not be realistic though and one needs more precise extractions of the pion FFs at moderately high- $p_{t}\left(p_{t}=1 \sim 3 \mathrm{GeV} / \mathrm{c}\right)$ before definite conclusions can be drawn. The fact that some standard PDFs and FFs fail to give a good description at forward rapidities may find a natural explanation in the observation that these data are sensitive to small- $x$ effects in the target.

Hence, we would like to analyze these high-energy, forward processes at RHIC within another theoretical approach, namely the "Color Glass Condensate" (CGC) formalism. Contrary to the DGLAP approach, it resums small- $x$ effects in the gluon distribution function. The Sivers functions, on the other hand, are unaffected as the SSA arises from large- $x$ valence quarks on the projectile side.

The CGC approach is most commonly applied to the study of gluon saturation effects at sufficiently small $x$ and/or $Q^{2}$ [15, 31]. At a given $x$, the transverse momentum below which the unintegrated gluon distribution saturates due to non-linear terms in the QCD evolution equations is called the saturation momentum $Q_{s}(x)$. It grows approximately as a power of energy [32, 33]; NLO BFKL evolution gives $Q_{s}(x) \sim 1 / x^{\lambda}$ with $\lambda \simeq 0.3[34]$. HERA phenomenology indicates that $Q_{s} \sim 1 \mathrm{GeV}$ at $x \sim 10^{-4}[11]$.

The deep saturation (non-linear) regime is therefore of minor relevance to semi-hard $\left(p_{t} \gtrsim 1 \mathrm{GeV}\right)$ particle production in $p p$ collisions at RHIC energy. This will be verified in a more quantitative way in section IV]

\footnotetext{
1 We note that these expressions are understood to apply at a fixed (average) scale. The scale dependence is not known yet and will not be considered here.
} 
However, the CGC formalism is also applicable to the so-called "extended" geometric scaling (EGS) regime 32, 35] which emerges above the saturation line up to transverse momenta of about $Q_{g s}=Q_{s}^{2} / \Lambda \gg Q_{s}$, where $\Lambda$ is a nonperturbative soft scale expected to be of order $\Lambda_{\mathrm{QCD}}$. In deep inelastic scattering (DIS) geometric scaling implies that at low $x(\lesssim 0.01)$ the proton structure function depends only on the scaling variable $\tau=Q^{2} / Q_{s}^{2}(x)$ rather than on $Q^{2}$ and $x$ separately.

Fits to DIS data from HERA [12, 13] found that approximate geometric scaling holds not only at small $Q^{2}$, where this is expected due to saturation effects, but over a much broader window $Q_{s}^{2}<Q^{2}<Q_{g s}^{2}$, thereby providing evidence for the existence of such a new regime. It arises if the solution of the LO-BFKL evolution equation is expanded to second order around the saturation saddle point 13 . The first term gives geometric scaling while the second "diffusion" term contributes to scaling violations ${ }^{2}$. Due to this diffusion term, the anomalous dimension $\gamma$ governing the small- $x$ evolution of the gluon distribution with $\operatorname{rapidity~} y=\log (1 / x)$ is shifted from $\gamma_{s} \simeq 0.63$ (the BFKL saddle point in the vicinity of the saturation line) by $\Delta \gamma \propto \log \left(1 / r_{t} Q_{s}\right) / y$, where $r_{t} \sim 1 / Q$ denotes the dipole size. As $Q$ approaches $\sim Q_{g s}$ from below, violations of geometric scaling grow to order unity and the anomalous dimension reaches $\gamma_{\text {DGLAP }} \sim 1$ (more precisely, $\left.1-\gamma_{\text {DGLAP }}=\mathcal{O}\left(\alpha_{s}\right)\right)$. Ref. 13 ] finds that the average shift required for a good fit to the HERA data is not small, $\langle\Delta \gamma\rangle \simeq 0.2$. Thus, the behavior of the anomalous dimension which determines the (geometric) scaling violations plays a key role for high-energy ep scattering.

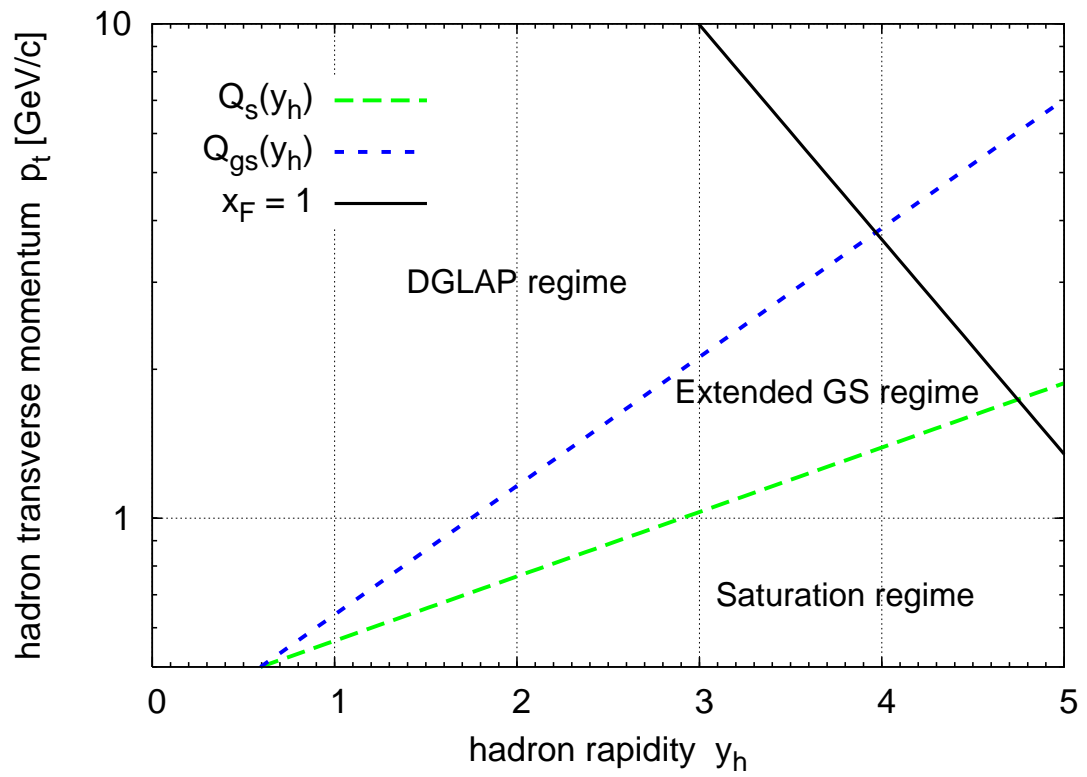

FIG. 2: "Phase diagram" of the target proton at RHIC energy $(\sqrt{s}=200 \mathrm{GeV}) \cdot y_{h}$ and $p_{t}$ are the rapidity and the transverse momentum of the produced hadron, respectively. The dashed line depicts the boundary $Q_{s}\left(y_{h}\right)$ of the saturation region; the dotted line that of extended geometric scaling, $Q_{g s}\left(y_{h}\right)$. For this plot we fixed the projectile parton's momentum fraction to the typical value $x_{1}=0.1$. The solid line corresponds to the boundary of phase space, $x_{F}=1$.

The same approach has also been applied to high- $p_{t}(\gtrsim 1 \mathrm{GeV})$ hadron production in $d A u$ collisions at RHIC over a wide interval of rapidity $y_{h}[17]$. Indeed, the data was shown to agree rather well with a parameterization of the anomalous dimension which increases logarithmically with $p_{t}$ from $\gamma=\gamma_{s}$ to its asymptotic value $\gamma \sim 1$, while decreasing with $y$ as $\Delta \gamma \sim 1 / y$ at very large rapidity. The $d A u$ data from RHIC is therefore consistent with

2 To find $Q_{g s} \sim Q_{s}^{2} / \Lambda$ one should in fact estimate $Q_{g s}$ from the transition point between the LLA and DLA saddle points, respectively, rather than from the diffusion term in the expansion of LO-BFKL about the saturation saddle point. For details see, for example, section 2.4.3 in ref. [15] and references therein. 
the presence of an extended geometric scaling regime above the saturation region which widens towards forward rapidities (i.e. smaller $x_{2}$ ). The EGS window is characterized by the need to resum logarithms of $1 / x_{2}$ rather than of $Q^{2}$. However, the twist expansion remains valid, all-twist resummation being necessary only in the saturation regime $Q^{2} \lesssim Q_{s}^{2}$.

We illustrate the various kinematic regimes emerging in $p p$ collisions at RHIC in Fig. 2 The boundaries of these regions were determined as follows. The saturation regime of the target proton extends to

$$
Q_{s}\left(x_{2}\right)=1 \mathrm{GeV}\left(\frac{x_{0}}{x_{2}}\right)^{\lambda / 2},
$$

where, as already referred to above, $x_{0}=3.0 \times 10^{-4}$ and $\lambda=0.3$ [1]. $x_{2}$ is related to the rapidity $y_{h}$ of the produced hadron by kinematics: $x_{1} / x_{2}=\exp \left(2 y_{h}\right)$ (see, for example, appendix B in [16]). For this figure, we fixed $x_{1}$ to a value typical for a valence quark, $x_{1}=10^{-1}$. The EGS regime corresponds to the window between $Q_{s}$ and $Q_{g s}$, where the anomalous dimension $\gamma$ (given below in eq. (15)) grows from $\gamma_{s}$ to $\sim 1$. In fig. 22 its upper limit is taken to be $Q_{g s}=Q_{s}^{2} / \Lambda$ with $\Lambda=0.5 \mathrm{GeV}$, for illustration. Above $Q_{g s}, \gamma \sim \gamma_{\text {DGLAP }}$ and so the DGLAP approximation applies; note that this of course includes the DLL regime of large $y_{h}$ and large $p_{t}$. The boundary of phase space, finally, is reached when the Feynman- $x$ of the produced hadron $x_{F}=\left(p_{t} / \sqrt{s}\right) \exp \left(y_{h}\right)=1$.

This figure confirms that the all-twists saturation regime plays no prominent role at RHIC energy and $p_{t} \gtrsim 1$ - $1.5 \mathrm{GeV} / \mathrm{c}$, regardless of rapidity. However, for $y_{h} \gtrsim 3$ the EGS window opens wide and eventually covers essentially the entire semi-hard regime of particle production as the DGLAP region is cut off by energy-momentum conservation constraints. This implies that at such rapidities, $\gamma$ does not reach 1 at any accessible transverse momentum. It may therefore be plausible that small- $x$ effects are relevant for semi-hard particle production in the forward region of $p p$ collisions at RHIC.

Forward particle production leads to a strong asymmetry in the momentum fractions $x_{1} \sim 1$ and $x_{2} \ll 1$ which are probed in the projectile and target, respectively. The process can then be described using an asymmetric DGLAP $\otimes$ CGC factorization scheme developed in ref. [16],

$$
\begin{aligned}
\frac{d N_{h}}{d y_{h} d^{2} p_{t}}= & \frac{1}{(2 \pi)^{2}} \int_{x_{F}}^{1} d x_{1} \frac{x_{1}}{x_{F}}\left[f_{q / p}\left(x_{1}, p_{t}^{2}\right) N_{F}\left(\frac{x_{1}}{x_{F}} p_{t}, x_{2}\right) D_{h / q}\left(\frac{x_{F}}{x_{1}}, p_{t}^{2}\right)\right. \\
& \left.+f_{g / p}\left(x_{1}, p_{t}^{2}\right) N_{A}\left(\frac{x_{1}}{x_{F}} p_{t}, x_{2}\right) D_{h / g}\left(\frac{x_{F}}{x_{1}}, p_{t}^{2}\right)\right]
\end{aligned}
$$

The indices $q$ in $f_{q / p}$ and $D_{h / q}$ are implicitly summed over all participating quark flavors. In what follows, the mass of the produced hadrons is neglected because $m_{\pi} \ll p_{t}$, which allows us to equate to a good approximation the pseudorapidity $\eta$ with the rapidity $y_{h}$.

The approach (13) includes DGLAP evolution of the projectile parton distribution functions $f_{q / p}\left(x_{1}, p_{t}^{2}\right)$ and of the fragmentation functions $D_{h / q}\left(x_{F} / x_{1}, p_{t}^{2}\right)$ with the factorization scale $Q^{2}=p_{t}^{2}$. It resums small- $x$ logarithms in the target as well as DGLAP logarithms of $Q^{2}$ on the projectile side and has been derived to leading order in $\alpha_{s}$ [16]. Exact rather than small- $x$ approximated splitting functions are to be employed, such that the projectile momentum is conserved, which is important at large $x_{1}$. On the other hand, the target proton is described by the dipole forward scattering amplitudes $N_{F, A}$, where $N_{F}$ corresponds to a projectile quark impinging on the target's small- $x$ gluons, while $N_{A}$ applies to a projectile gluon. These arise [36] as averages of two-point functions of Wilson lines in the corresponding representation (running up and down the light-cone, separated by a transverse distance $r_{t}$ ) over the gluon field of the hadron [37]. In the high-energy limit one therefore recovers Mueller's dipole approach [38]. For a recent comparison of this approach with HERA diffractive electroproduction data we refer to ref. [39].

The dipole profile employed here is similar to that from the fit presented in ref. 11] to HERA DIS data, except for the presence of the anomalous dimension $\gamma<1$ [13, 14, 15, 16, 17]:

$$
N_{A}\left(r_{t}, x_{2}\right)=1-\exp \left[-\frac{1}{4}\left(r_{t}^{2} Q_{s}^{2}\left(x_{2}\right)\right)^{\gamma\left(r_{t}, x_{2}\right)}\right] .
$$


$r_{t}$ denotes the transverse size of the dipole. $N_{F}$ is obtained here by the replacement $Q_{s}^{2} \rightarrow\left(C_{F} / C_{A}\right) Q_{s}^{2}=$ $(4 / 9) Q_{s}^{2}$, corresponding to a weaker coupling of a quark relative to a gluon projectile to the target field. Due to this color factor, our effective saturation momentum entering $N_{F}$ is somewhat smaller than the original GolecBiernat-Wüstoff (GB-W) fit 11] but agrees with previous comparisons to RHIC data [14, 16, 17] and with the analysis of HERA DIS data presented in ref. [13].

The Ansatz (14) exhibits saturation at $r_{t}>1 / Q_{s}$ and also reproduces the $N_{A} \sim r_{t}^{2}$ DGLAP limit as $r_{t} \rightarrow 0$ (assuming that also $\gamma \rightarrow 1$ in that limit) $)^{3}$. It also displays exact geometric scaling when $\gamma$ is constant since then $N\left(r_{t}, x\right)$ depends only on the scaling variable $\rho \equiv r_{t} Q_{s}$. Violations of geometric scaling arise due to the dependence of $\gamma$ on $r_{t}$, increasing from $\gamma_{s}$ at $r_{t} \sim 1 / Q_{s}$ to $\gamma_{\text {DGLAP }} \sim 1$ at very short distances. Here, the anomalous dimension $\gamma\left(r_{t}, x_{2}\right)$ of the gluon distribution is parameterized as

$$
\gamma\left(r_{t}, x_{2}\right)=\gamma_{s}+\left(1-\gamma_{s}\right) \frac{\log \left(1 / r_{t}^{2} Q_{s}^{2}\left(x_{2}\right)\right)}{\lambda y_{2}+d \sqrt{y_{2}}+\log \left(1 / r_{t}^{2} Q_{s}^{2}\left(x_{2}\right)\right)}
$$

with $\gamma_{s} \simeq 0.627 ; y_{2}=\log 1 / x_{2}$ is (minus) the rapidity of the target partons. This function agrees with the solution of LO-BFKL evolution with saturation boundary conditions in the saddle point approximation [13], and with the general theoretical limits mentioned above. However, the parameterization (15) also includes subleading corrections $\sim d \sqrt{y_{2}}$ which govern geometric scaling violations at subasymptotic rapidities. With $d \simeq 1.2$ the anomalous dimension (15) was shown to provide a good description of $d A u$ data from RHIC over a wide range of rapidity [17]. We note that (15) applies only in the EGS regime, while $\gamma=\gamma_{s}$ stays fixed within the saturation region (which is, however, not important here, see section IV).

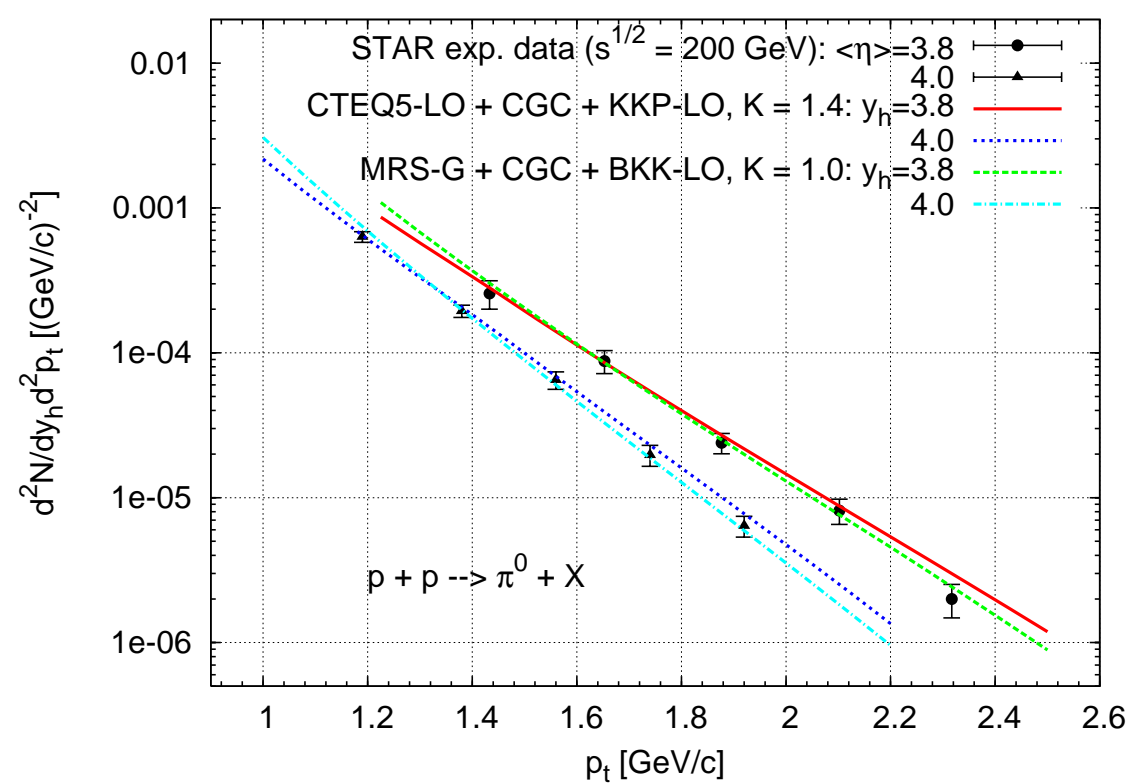

FIG. 3: Transverse momentum distributions of forward inclusive $\pi^{0}$ from unpolarized $p p$ collisions at $\sqrt{s}=200 \mathrm{GeV}$. The STAR data 29] were taken at average pseudorapidities of $\langle\eta\rangle=3.8$ and 4.0, respectively. Theoretical results correspond to two different sets of parton distribution and fragmentation functions, i.e. CTEQ5-LO+KKP-LO (I) and MRS-G+BKK-LO (II), respectively, all evaluated at $Q^{2}=p_{t}^{2}$. They give $\chi^{2}$ per data point of $0.9(0.3)$ at $y=3.8$ and $0.7(1.0)$ at $y=4.0$ for set I (II).

${ }^{3}$ Note that in order to reproduce the $\sim 1 / q_{t}^{4}$ DGLAP power-law tail in momentum space one needs to introduce an additional logarithm of $1 / r_{t}^{2}$ in the exponent of [14. For $\gamma=1, N_{A}\left(q_{t}\right)$ then drops approximately exponentially in the high-density regime about $q_{t} \sim Q_{s}$, turning into $\sim 1 / q_{t}^{4}$ LO DGLAP behavior as $q_{t} \rightarrow \infty$ [19, 40]. A similar modification of the GB-W model was considered in ref. [41]. 
To determine the transverse momentum distribution of hadrons via eq. (13) requires taking the Fourier transforms of the dipole profiles (14) to momentum space. To simplify this task, we shall replace $\gamma\left(r_{t}, x_{2}\right)$ by $\gamma\left(1 / q_{t}, x_{2}\right)$, where $q_{t}=\left(x_{1} / x_{F}\right) p_{t}$ is the Fourier conjugate of $r_{t}$. This provides a good approximation at large $y_{h}$ (small $x_{2}$ ) since there $\gamma$ increases rather slowly with $q_{t}$.

In fig. 3 we present our numerical results for the $p_{t}$-distributions of forward inclusive pions from $p p$ collisions at $\sqrt{s}=200 \mathrm{GeV}$. They correspond to rapidities $y_{h}=3.8$ and 4.0 , respectively. We employ two different sets of parton distribution $(\mathrm{PDF})$ and fragmentation $(\mathrm{FF})$ functions: CTEQ5-LO PDFs [2] and KKP-LO FFs [3], which is nowadays a common combination. The other set is MRS-G PDFs [44] and BKK-LO FFs [45] as used in ref. 20] to extract the parameterization of the Sivers functions from experimental data obtained at lower energies. We observe rather good agreement of both sets in the interval $1.2 \lesssim p_{t} \lesssim 2.4 \mathrm{GeV} / \mathrm{c}$ with recent STAR data from RHIC [29]. For the overall normalization, we need to multiply our LO results by a factor $K \simeq 1.4$ for the CTEQ5-LO+KKP-LO set I, while $K \simeq 1.0$ for the MRS-G+BKK-LO set II. The $\chi^{2}$ per data point is 0.9 $(0.3)$ at $y=3.8$ and $0.7(1.0)$ at $y=4.0$ for set I (II), which is rather good. Since both sets work equally well within error bars, we will henceforth employ set II which does not require a K-factor.

The results from fig. 3 correspond to a scale $Q^{2}=p_{t}^{2}$. The scale dependence in eq. (13) arises entirely from the LO PDFs and FFs and amounts to a weakly $p_{t}$-dependent normalization factor [16]. If the scale dependence of the LO Sivers functions turns out to be similar to that of the unpolarized PDFs, such an overall $K$-factor would cancel in the ratio of the polarized to unpolarized processes. The evolution properties of the Sivers functions are, however, largely unknown at present and as a consequence we have to postpone a more detailed study of such issues to the future.

\section{B. $\quad p^{\uparrow} p$ collisions and single transverse-spin asymmetry}

The single-inclusive pion distribution of eq. (13) is written in a collinearly factorized convolution form, $d \sigma(p p \rightarrow$ $h X) \sim f_{q / p} \otimes N_{F} \otimes D_{h / q}$. We shall formulate the single transverse-spin asymmetry $A_{N}$ in an analogous way but partly include transverse momentum dependence in order to incorporate the Sivers effect. For the numerator of eq. (11) this results in

$$
\begin{aligned}
d \sigma\left(p^{\uparrow} p \rightarrow h X\right)-d \sigma\left(p^{\downarrow} p \rightarrow h X\right) & \propto \int d^{2} k_{t}\left[f_{q / p^{\uparrow}}\left(x_{1}, \vec{k}_{t}\right)-f_{q / p^{\downarrow}}\left(x_{1}, \vec{k}_{t}\right)\right] \otimes N_{F}\left(x_{1}, \vec{q}_{t}, \vec{k}_{t}\right) \otimes D_{h / q}\left(x_{F} / x_{1}\right) \\
& \simeq \Delta^{N} f_{q / p^{\uparrow}}\left(x_{1}\right) \otimes\left[N_{F}\left(x_{1}, q_{t}-k_{t}^{0}\right)-N_{F}\left(x_{1}, q_{t}+k_{t}^{0}\right)\right] \otimes D_{h / q}\left(x_{F} / x_{1}\right),(10
\end{aligned}
$$

where we used eq. (6) to arrive at the last line. Also, we simplified the kinematics (as was done in refs. 20] and therefore has to be considered as part of the fit of the Sivers function) by picking up only contributions where $\vec{k}_{t}$ is either parallel or anti-parallel to $\vec{q}_{t}$.

On the other hand, the denominator of (1) is given by

$$
\begin{aligned}
d \sigma\left(p^{\uparrow} p \rightarrow h X\right)+d \sigma\left(p^{\downarrow} p \rightarrow h X\right) & \propto \int d^{2} k_{t}\left[f_{q / p^{\uparrow}}\left(x_{1}, \vec{k}_{t}\right)+f_{q / p^{\downarrow}}\left(x_{1}, \vec{k}_{t}\right)\right] \otimes N_{F}\left(x_{1}, \vec{q}_{t}, \vec{k}_{t}\right) \otimes D_{h / q}\left(x_{F} / x_{1}\right) \\
& \simeq 2 f_{q / p}\left(x_{1}\right) \otimes N_{F}\left(x_{1}, q_{t}\right) \otimes D_{h / q}\left(x_{F} / x_{1}\right)
\end{aligned}
$$

where eq. (4) and the fact that $f_{q / p}\left(x, \vec{k}_{t}\right)$ is an even function of $\vec{k}_{t}$ have been used. Thus, when displaying explicitly all arguments of the various functions the asymmetry becomes

$$
A_{N}\left(p_{t}, y_{h}\right)=\frac{1}{2} \frac{\sum_{\text {val }-q} \Delta^{N} f_{q / p^{\uparrow}}\left(x_{1}\right) \otimes\left[N_{F}\left(\frac{x_{1}}{x_{F}} p_{t}-k_{t}^{0}, y_{2}\right)-N_{F}\left(\frac{x_{1}}{x_{F}} p_{t}+k_{t}^{0}, y_{2}\right)\right] \otimes D_{h / q}\left(\frac{x_{F}}{x_{1}}, p_{t}^{2}\right)}{\sum_{q} f_{q / p}\left(x_{1}, p_{t}^{2}\right) \otimes N_{F}\left(\frac{x_{1}}{x_{F}} p_{t}, y_{2}\right) \otimes D_{h / q}\left(\frac{x_{F}}{x_{1}}, p_{t}^{2}\right)+f_{g / p}\left(x_{1}, p_{t}^{2}\right) \otimes N_{A}\left(\frac{x_{1}}{x_{F}} p_{t}, y_{2}\right) \otimes D_{h / g}\left(\frac{x_{F}}{x_{1}}, p_{t}^{2}\right)} .
$$

Only valence-quark contributions are accounted for in the numerator, according to the parameterization of the Sivers functions from ref. 20]. On the other hand, the unpolarized cross section in the denominator includes 
the contributions of all active quark flavors and of gluons. At large rapidities, however, it is also dominated by valence quarks. It is evident from (18) that a good description of the elementary quark $q_{t}$ distribution and of its derivative is required to reliably extract the Sivers function from $A_{N}$.

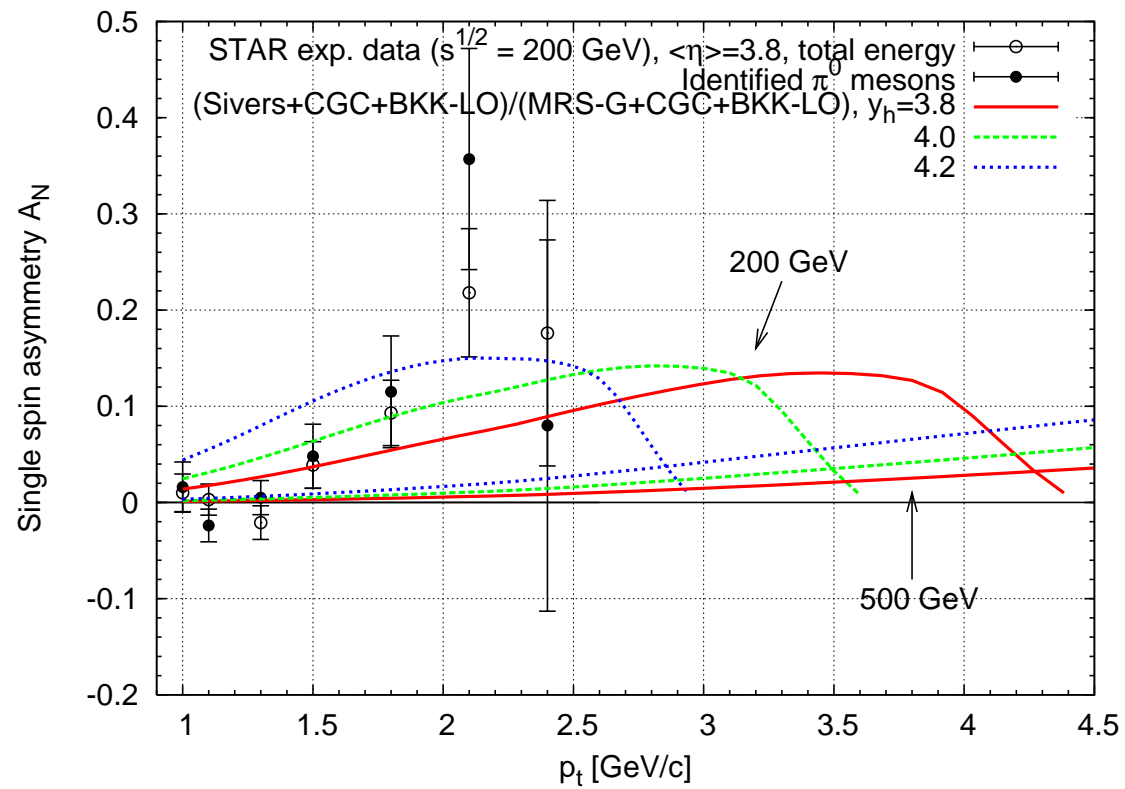

FIG. 4: Single transverse-spin asymmetry $A_{N}$ in the rapidity interval $y_{h}=3.8-4.2$ for $\sqrt{s}=200,500 \mathrm{GeV}$, with $K_{\Delta f}=2$ (two times larger Sivers functions than ref. [20]). The STAR data [7] were taken at an average pseudorapidity of $\langle\eta\rangle=3.8$ and $\sqrt{s}=200 \mathrm{GeV}$.

In Fig. 4 we compare our numerical results for the asymmetry $A_{N}$ to STAR data 7] taken at $\sqrt{s}=200 \mathrm{GeV}$. We also show predictions for larger rapidities, $y_{h}=4.0$ and 4.2 , and for higher energy, $\sqrt{s}=500 \mathrm{GeV}$. The theoretical curves were obtained from eq. (18) with the MRS-G [44] PDFs for the unpolarized projectile proton and the BKK-LO 45] FFs, the dipole (14), the anomalous dimension (15), and the saturation momentum (12). For the Sivers functions, we adopted the parameterization (91011) with $K_{\Delta f}=2$, corresponding to a two times larger Sivers effect as compared to the original fit from ref. [20].

One observes that our curve at $y_{h}=3.8$ goes up more slowly than the data and peaks at large $p_{t}$ around $p_{t} \sim 3.5 \mathrm{GeV} / \mathrm{c}$. At higher rapidity, however, the curves increase more steeply and the peak shifts to smaller $p_{t}$. At fixed rapidity, the asymmetry decreases rather rapidly with energy. This is mainly a kinematic effect in that the typical momentum fraction $x_{1}$ of projectile partons decreases, and so does the Sivers function (9). Given the theoretical uncertainty in the Sivers functions and the sizable errors of the data, the agreement can be considered reasonable. Even with the present large error bars, however, the STAR data appear to imply larger Sivers functions than previously thought [20] (as reflected by $K_{\Delta f}=2$ ), at least if $A_{N}$ indeed arises entirely from the Sivers effect. If more precise data becomes available in the future, our formalism (extended to allow for more general $\vec{k}_{t}$ dependence of the Sivers functions if needed) could be used for a complete refit of the Sivers functions.

A few additional comments regarding the factor $K_{\Delta f} \gtrsim 2$ found here are in order. The parameterization (9 11) of the Sivers function resulted from a fit to low-energy fixed-target data, for which an accurate description of the pion transverse momentum distribution (and of its slope) is hard to obtain. It is therefore not entirely surprising that the new high-energy data, while confirming qualitative features of the parameterizations, may still require some quantitative adjustments. Of course, such adjustments would alter predictions for asymmetries of other processes, like for instance the Drell-Yan process 22]. When high energy data with smaller error bars becomes available we intend to perform a more detailed investigation of the $x$ - and $\vec{k}_{t}$-dependence of the Sivers functions, rather than of their overall normalization only.

We should also point out that a good description of the STAR data can also be achieved by increasing the 
intrinsic transverse momentum $k_{t}(x)$ of valence quarks substantially, as recent parameterizations show [46]. This is because the Sivers distribution (9) is proportional to the intrinsic transverse momentum, and another factor of $k_{t}$ arises in eq. (16) from the difference of the contributions of quarks with $\vec{k}_{t}$ parallel or anti-parallel to the momentum transfer; see for example eq. (23) below. However, the process considered here is dominated by large $x$ on the projectile side $\left(x_{1} \sim 0.1-1\right)$, where intrinsic transverse momenta should probably not exceed the inverse proton radius by much. This is consistent also with our finding (and that from NLO DGLAP approaches) that the forward inclusive pion distribution from unpolarized collisions can be described rather well without assuming large intrinsic transverse momenta of projectile valence quarks.

\section{HIGHER-TWIST EFFECTS ON SSA}

The unitarized dipole profiles $N_{F, A}$ entering (1617) resum all higher-twist effects which arise at low transverse momentum. It is interesting to disentangle the leading-twist contribution to the observables considered here. For $p_{t}>Q_{s}$, one expects that higher-twist contributions are suppressed.

We estimate the leading contribution by introducing

$$
N_{F}\left(r_{t}, y_{2} ; c\right) \equiv \frac{1}{c}\left\{1-\exp \left[-\frac{c}{4}\left(4 r_{t}^{2} Q_{s}^{2}\left(y_{2}\right) / 9\right)^{\gamma\left(r_{t}, y_{2}\right)}\right]\right\}
$$

For $c=1$ we recover the full $N_{F}\left(r_{t}, y_{2}\right)$ from (14). In the limit $c \rightarrow 0$, however, the Fourier transform of (19) isolates the leading contribution in the expansion with respect to $Q_{s} / q_{t}$. For $q_{t} \neq 0$ one obtains

$$
\begin{aligned}
N_{F}\left(q_{t} \neq 0, y_{2} ; c\right) & =-\int d^{2} r_{t} e^{i \vec{q}_{t} \cdot \vec{r}_{t}} N_{F}\left(r_{t}, y_{2} ; c\right) \\
& =\frac{2 \pi}{c} \int_{0}^{\infty} d r_{t} r_{t} J_{0}\left(r_{t} q_{t}\right) \exp \left[-\frac{c}{4}\left(4 r_{t}^{2} Q_{s}^{2}\left(y_{2}\right) / 9\right)^{\gamma\left(r_{t}, y_{2}\right)}\right]
\end{aligned}
$$

We know analytic forms of the Fourier transform (20) only for $\gamma=1 / 2$ and 1 [16]. In the limit $c \rightarrow 0$ one finds

$$
\begin{aligned}
N_{F}^{\gamma=1 / 2}\left(q_{t}, y_{2} ; c\right) & =\frac{\pi Q_{s}}{3\left[\left(Q_{s} c / 6\right)^{2}+q_{t}^{2}\right]^{3 / 2}} \stackrel{c \rightarrow 0}{\longrightarrow} \frac{\pi}{3 Q_{s}^{2}}\left(\frac{Q_{s}}{q_{t}}\right)^{3}, \\
N_{F}^{\gamma=1}\left(q_{t}, y_{2} ; c\right) & =\frac{9 \pi}{Q_{s}^{2} c^{2}} \exp \left[-\frac{9}{4 c}\left(\frac{q_{t}}{Q_{s}}\right)^{2}\right] \stackrel{c \rightarrow 0}{\longrightarrow} 0 .
\end{aligned}
$$

Hence, for $\gamma=1 / 2$, representing the LO-BFKL anomalous dimension without saturation boundary conditions, the Fourier transformed dipole profile exhibits a leading-twist tail $\sim\left(Q_{s} / q_{t}\right)^{3}$ (we recall that $\sim\left(Q_{s} / q_{t}\right)^{4}$ emerges in the DGLAP regime). On the other hand, the GB-W model [11] corresponding to $\gamma=1$ shows no power-law tail at large $q_{t}$. It arises entirely from all-twist resummation and has no expansion in powers of $Q_{s} / q_{t}$.

For the anomalous dimension from (15) the Fourier transform and its $c \rightarrow 0$ limit cannot be obtained analytically. We therefore show numerical results in Fig. [5] Here, we set $y_{h}=3.8, p_{t}=2 \mathrm{GeV} / \mathrm{c}$, which represent typical values, and take $x_{1}=x_{F}$ such that $p_{t}=q_{t}$.

One observes that the profile for $\gamma=1 / 2$ is almost constant in the interval $0 \leq c \leq 1$ and approaches a finite value as $c \rightarrow 0$, which agrees with the analytic result (21). This confirms that for these kinematic conditions the dipole with anomalous dimension from LO-BFKL without saturation boundary condition is dominated entirely by the leading-twist contribution. Next, we consider the $q_{t}$-dependent anomalous dimension (15) which generates violations of geometric scaling and interpolates to the DGLAP regime at high $q_{t}$. For this case, the Fourier transformed dipole profile decreases slowly as $c \rightarrow 0$ from its value at $c=1$. This decrease corresponds to highertwist contributions of about $8 \%$. Thus, higher-twist contributions to single-inclusive forward hadron production in $p p$ collisions are not very large once an anomalous dimension with the described features is taken into account. Quantitative comparisons to the anomalous dimension from leading-twist NLO DGLAP approaches for the present kinematics would be interesting. 


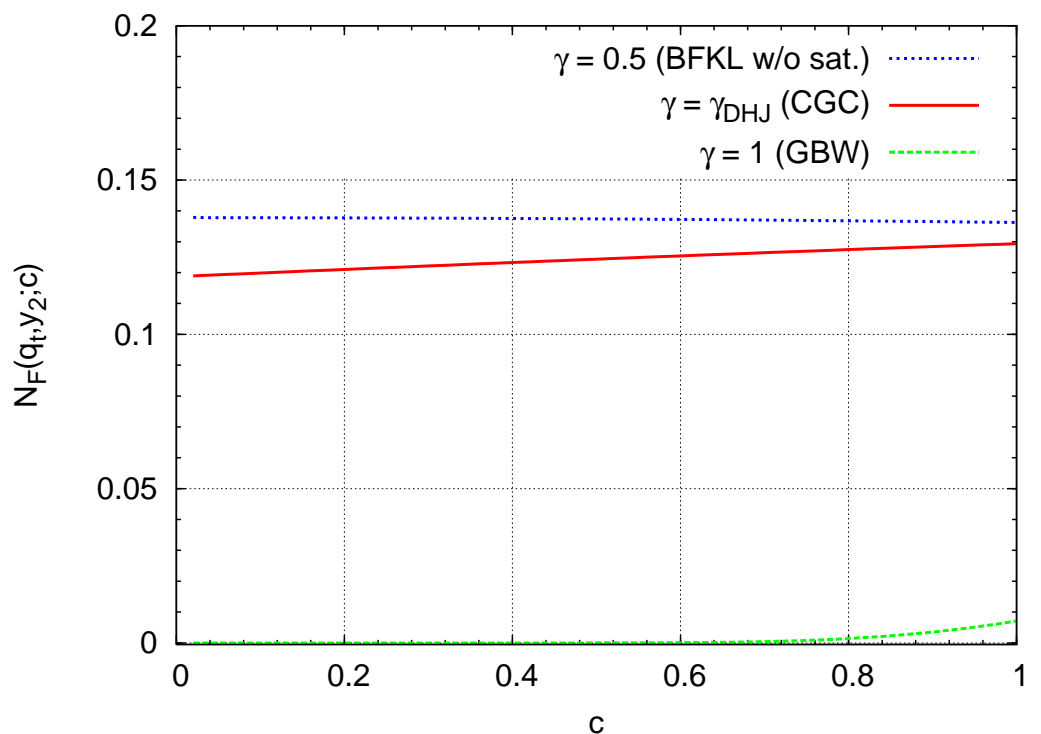

FIG. 5: Study of leading and higher twist contributions in the dipole profile $N_{F}\left(q_{t}, y_{2}\right)$. We plot the results of eq. (20) with $\gamma=0.5,1$ and $\gamma_{\mathrm{DHJ}}$ from (15), respectively, as a function of $c$ at $y_{h}=3.8, p_{t}=2 \mathrm{GeV} / \mathrm{c}$ and $x_{1}=x_{F}$.

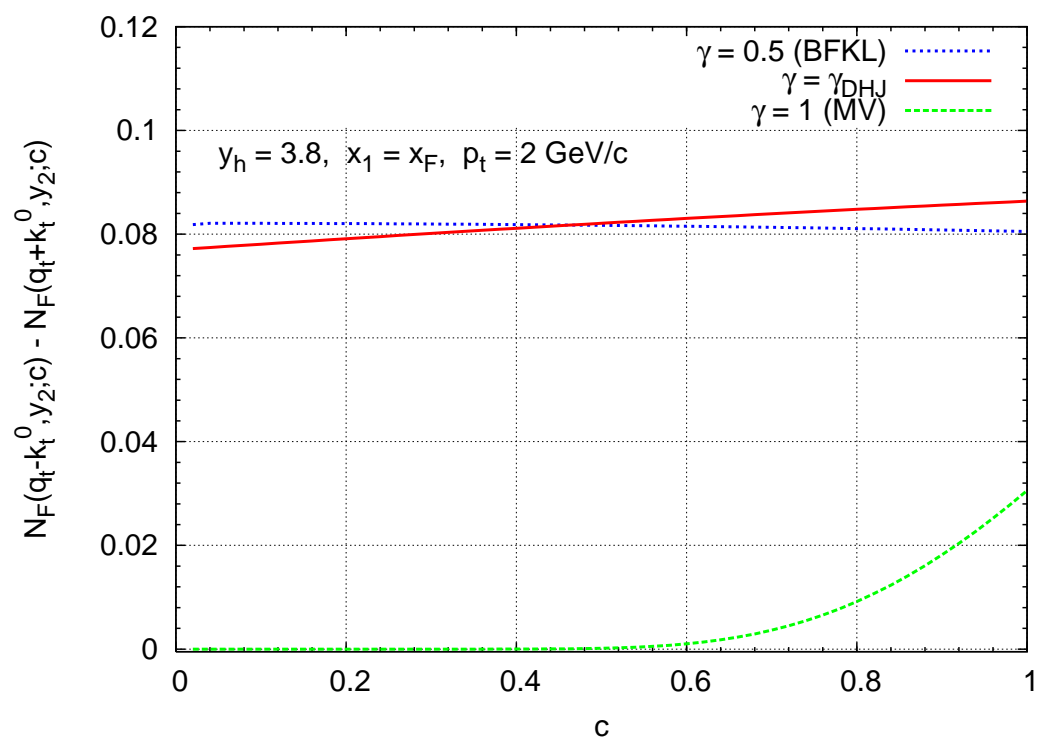

FIG. 6: Same as Fig. 5 but now for the difference $N_{F}\left(q_{t}-k_{t}^{0}\right)-N_{F}\left(q_{t}+k_{t}^{0}\right)$.

Next, we address the same question for the difference appearing in the numerator of the asymmetry $A_{N}$ which essentially corresponds to the derivative of $N_{F}\left(q_{t}\right)$. For this quantity, the leading contribution is suppressed by an additional power of $k_{t}^{0} / q_{t}$ as can be easily realized by considering the leading contribution for $\gamma=1 / 2$ as an example:

$$
\frac{Q_{s}^{3}}{\left(q_{t}-k_{t}^{0}\right)^{3}}-\frac{Q_{s}^{3}}{\left(q_{t}+k_{t}^{0}\right)^{3}}=6 \frac{Q_{s}^{3}}{q_{t}^{3}} \frac{k_{t}^{0}}{q_{t}}+\cdots
$$


Due to this additional factor, the magnitude of $N_{F}\left(q_{t}-k_{t}^{0}\right)-N_{F}\left(q_{t}+k_{t}^{0}\right)$ as shown in fig. [6 is smaller than that of $N_{F}\left(q_{t}\right)$ from fig. 5 Here, too, the $c \rightarrow 0$ limit isolates the leading power of $Q_{s} / q_{t}$ from this quantity. The curve for $\gamma=1 / 2$ is again flat over the entire interval, implying leading-twist dominance. On the other hand, for $\gamma=1$ (GB-W) the leading-twist contribution vanishes completely. For the $q_{t}$-dependent anomalous dimension (15) we observe higher-twist contributions of about $11 \%$.

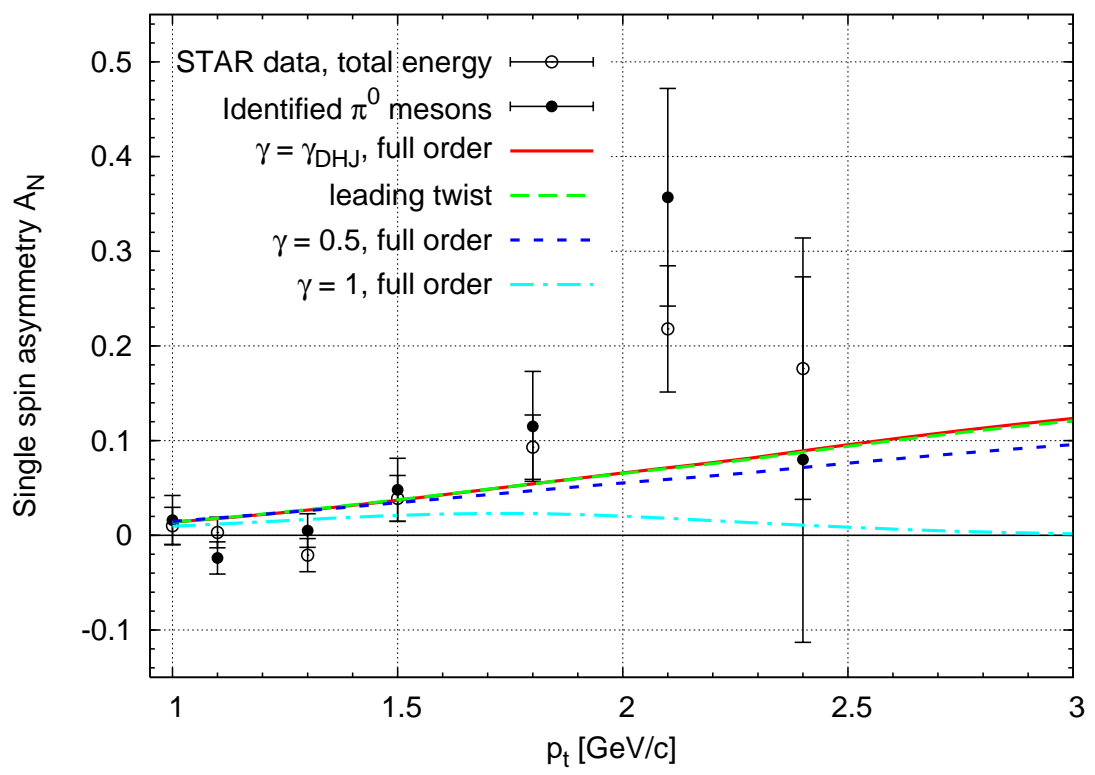

FIG. 7: The influence of the anomalous dimension on the asymmetry $A_{N}$ at $y_{h}=3.8$ and $1 \leq p_{t} \leq 3 \mathrm{GeV} / \mathrm{c}$.

Finally, Fig. [7 depicts the asymmetry $A_{N}$ over the range $1 \leq p_{t} \leq 3 \mathrm{GeV} / \mathrm{c}$ at $y_{h}=3.8$ and $\sqrt{s}=200 \mathrm{GeV}$ for $\gamma=1 / 2,1$, and $\gamma=\gamma_{\mathrm{DHJ}}$ from eq. 15). For the latter case, we also show $A_{N}$ as obtained from the leadingtwist contribution to $N_{F}$ alone. Since the full and leading-twist curves are almost identical, we conclude that higher-twist contributions in the numerator and denominator cancel to a large extent. On the other hand, it turns out that the growth of the anomalous dimension with transverse momentum (geometric scaling violations) does give rise to a slightly steeper $A_{N}\left(p_{t}\right)$ as compared to a constant $\gamma=1 / 2$. The GB-W dipole, which does not permit a twist expansion, gives a very small asymmetry. The reason for this behavior is the unrealistically large contribution from gluons to the unpolarized cross section in the denominator of $A_{N}$ at high rapidity: in momentum space, $N_{A}\left(q_{t}\right) \sim \exp \left[-q_{t}^{2} / Q_{s}^{2}\right]$ falls off less rapidly with $q_{t}$ than $N_{F}\left(q_{t}\right) \sim \exp \left[-\left(C_{A} / C_{F}\right) q_{t}^{2} / Q_{s}^{2}\right]$.

\section{SUMMARY}

In this paper, we have analyzed recent high-energy data from STAR on inclusive pion production at forward rapidities and on single transverse spin asymmetries (SSA) in $p^{\uparrow} p$ collisions. Particle production at large rapidities involves very small momentum fractions in the target, hence we propose and use a factorization approach which accounts for small- $x$ effects on the gluon distribution of the target. Within this "Color Glass Condensate" approach, the target is described by unitarized two-point functions of light-like Wilson lines (dipole forward scattering amplitudes) rather than the usual leading-twist, leading-log (DGLAP) gluon distribution function.

We have shown explicitly that the deeply non-linear ("saturation") regime is of little relevance for particle production in $p p$ collisions at RHIC energy. The dipole scattering amplitude is therefore well approximated by its leading-twist limit $N\left(r_{t}, x\right) \sim\left(r_{t} Q_{s}(x)\right)^{2 \gamma\left(r_{t}, x\right)}$. Nevertheless, small- $x$ evolution not only leads to saturation for transverse momenta $q_{t} \leq Q_{s}(x)$ but also to a so-called "extended geometric scaling" window above $Q_{s}$, which has been confirmed by fits to HERA DIS data. Within the EGS window extending from $Q_{s}(x)$ to $Q_{g s}(x) \sim Q_{s}^{2}(x) / \Lambda \gg$ 
$Q_{s}(x)$, the anomalous dimension $\gamma$ of the gluon distribution function increases smoothly from its value at the BFKL saddle-point with saturation boundary conditions, $\gamma_{s} \simeq 0.63$, to its value in the dilute DGLAP regime, $\gamma_{\text {DGLAP }} \sim 1$. Once this feature is incorporated, a very good baseline for forward pion production in unpolarized $p p$ collisions is obtained $\left(\chi^{2}\right.$ per data point $\left.\approx 1\right)$. A comparison of our $\gamma$ to that from NLO DGLAP approaches in the kinematic regime relevant for STAR would be interesting, given that the predicted pion $p_{t}$-distributions are similar.

Building on this baseline, we then proceed to analyze single transverse-spin asymmetries in the forward region. We restrict ourselves to SSAs originating from the Sivers effect, which is nowadays believed to give the dominant contribution within the framework of transverse spin and transverse momentum dependent parton distribution functions. The approximately $10 \%$ contributions from higher twists to the polarized and unpolarized cross sections, respectively, largely cancel in their ratio $A_{N}$, which is therefore very well approximated by the leading-twist contribution. Furthermore, we find that a parameterization of the Sivers functions obtained previously from a fit to fixed-target $p p$ data [20] within the LO DGLAP approach provides a reasonable description of the high-energy STAR data, if their overall normalization is scaled up by at least a factor of two. This might indicate that the Sivers effect is significantly stronger than thought so far and that small- $x$ effects do not cancel in the ratio, by affecting the slope of the cross section. More quantitative statements about the Sivers functions must await data with smaller errors and over some range of energies.

\section{Acknowledgments}

We thank Werner Vogelsang for useful discussions regarding the DGLAP results, and Mauro Anselmino, Umberto D'Alesio and Francesco Murgia for valuable comments. The research of D.B. has been made possible by financial support from the Royal Netherlands Academy of Arts and Sciences and in addition, is part of the research programme of the 'Stichting voor Fundamenteel Onderzoek der Materie (FOM)', which is financially supported by the 'Nederlandse Organisatie voor Wetenschappelijk Onderzoek (NWO)'.

[1] D. L. Adams et al. [FNAL-E704 Collaboration], Phys. Lett. B 264, 462 (1991); Z. Phys. C 56, 181 (1992); K. Krueger et al., Phys. Lett. B 459, 412 (1999); V. V. Abramov et al., arXiv:hep-ex/0511036

[2] G. L. Kane, J. Pumplin and W. Repko, Phys. Rev. Lett. 41, 1689 (1978).

[3] D. W. Sivers, Phys. Rev. D 41, 83 (1990); ibid. D 43, 261 (1991).

[4] J. C. Collins, Nucl. Phys. B 396, 161 (1993).

[5] M. Anselmino, M. Boglione and F. Murgia, Phys. Lett. B 362, 164 (1995).

[6] J. w. Qiu and G. Sterman, Phys. Rev. D 59, 014004 (1999); D. Boer, Phys. Rev. D 60, 014012 (1999); Y. Koike, AIP Conf. Proc. 675, 449 (2003).

[7] J. Adams et al. [STAR Collaboration], Phys. Rev. Lett. 92, 171801 (2004).

[8] F. Videbaek [BRAHMS Collaboration], arXiv:nucl-ex/0601008

[9] C. Bourrely and J. Soffer, Eur. Phys. J. C 36, 371 (2004).

[10] L. C. Bland, arXiv:hep-ex/0602012

[11] K. Golec-Biernat and M. Wüsthoff, Phys. Rev. D 59, 014017 (1999); Phys. Rev. D 60, 114023 (1999).

[12] A. M. Stasto, K. Golec-Biernat and J. Kwiecinski, Phys. Rev. Lett. 86, 596 (2001); C. Marquet and L. Schoeffel, Phys. Lett. B 639, 471 (2006).

[13] E. Iancu, K. Itakura and S. Munier, Phys. Lett. B 590, 199 (2004).

[14] D. Kharzeev, Y.V. Kovchegov and K. Tuchin, Phys. Lett. B 599, 23 (2004).

[15] J. Jalilian-Marian and Y. V. Kovchegov, Prog. Part. Nucl. Phys. 56, 104 (2006).

[16] A. Dumitru, A. Hayashigaki and J. Jalilian-Marian, Nucl. Phys. A 765, 464 (2006).

[17] A. Dumitru, A. Hayashigaki and J. Jalilian-Marian, Nucl. Phys. A 770, 57 (2006).

[18] M. Anselmino, M. Boglione, U. D’Alesio, E. Leader and F. Murgia, Phys. Rev. D 71, 014002 (2005).

[19] D. Boer and A. Dumitru, Phys. Lett. B 556, 33 (2003).

[20] M. Anselmino and F. Murgia, Phys. Lett. B 442, 470 (1998).

[21] M. Anselmino, M. Boglione and F. Murgia, Phys. Rev. D 60, 054027 (1999).

[22] M. Anselmino, U. D'Alesio and F. Murgia, Phys. Rev. D 67, 074010 (2003). 
[23] A. Bacchetta, U. D’Alesio, M. Diehl and C. A. Miller, Phys. Rev. D 70, 117504 (2004).

[24] D. Boer and P. J. Mulders, Phys. Rev. D 57, 5780 (1998).

[25] J. C. Collins and D. E. Soper, Nucl. Phys. B 193, 381 (1981) [Erratum-ibid. B 213, 545 (1983)]; X. Ji, J. p. Ma and F. Yuan, Phys. Rev. D 71, 034005 (2005).

[26] J. C. Collins, Phys. Lett. B 536, 43 (2002); C. J. Bomhof, P. J. Mulders and F. Pijlman, Phys. Lett. B 596, 277 (2004).

[27] M. Anselmino et al., arXiv:hep-ph/0511017.

[28] M. Boglione and P. J. Mulders, Phys. Rev. D 60, 054007 (1999).

[29] J. Adams et al. [STAR Collaboration], nucl-ex/0602011

[30] V. Guzey, M. Strikman and W. Vogelsang, Phys. Lett. B 603, 173 (2004).

[31] for recent reviews see, for example, A. H. Mueller, Nucl. Phys. A 715, 20 (2003); E. Iancu and R. Venugopalan, hep-ph/0303204 L. McLerran, Nucl. Phys. A 752, 355 (2005); E. Levin, J. Phys. Conf. Ser. 5, 127 (2005) arXiv:hep-ph/0408039; L. Frankfurt, M. Strikman and C. Weiss, Ann. Rev. Nucl. Part. Sci. 55, 403 (2005).

[32] A. H. Mueller and D. N. Triantafyllopoulos, Nucl. Phys. B 640, 331 (2002).

[33] S. Munier and R. Peschanski, Phys. Rev. D 69, 034008 (2004); Phys. Rev. D 70, 077503 (2004).

[34] D. N. Triantafyllopoulos, Nucl. Phys. B 648, 293 (2003).

[35] E. Iancu, K. Itakura and L. McLerran, Nucl. Phys. A 708, 327 (2002); E. Levin and K. Tuchin, Nucl. Phys. A 691, 779 (2001).

[36] F. Gelis and J. Jalilian-Marian, Phys. Rev. D 67, 074019 (2003).

[37] I. Balitsky, Nucl. Phys. B 463, 99 (1996); Y. V. Kovchegov, Phys. Rev. D 60, 034008 (1999).

[38] A. H. Mueller, Nucl. Phys. B 415, 373 (1994); A. H. Mueller and B. Patel, Nucl. Phys. B 425, 471 (1994); E. Iancu and A. H. Mueller, Nucl. Phys. A 730, 460 (2004); E. Levin and M. Lublinsky, Phys. Lett. B 607, 131 (2005).

[39] J. R. Forshaw, R. Sandapen and G. Shaw, arXiv:hep-ph/0608161

[40] E. Iancu, K. Itakura and D. N. Triantafyllopoulos, Nucl. Phys. A 742, 182 (2004);

[41] J. Bartels, K. Golec-Biernat and H. Kowalski, Phys. Rev. D 66, 014001 (2002).

[42] H. L. Lai et al. [CTEQ Collaboration], Eur. Phys. J. C 12, 375 (2000).

[43] B. A. Kniehl, G. Kramer and B. Pötter, Nucl. Phys. B 582, 514 (2000).

[44] A. D. Martin, W. J. Stirling and R. G. Roberts, Phys. Lett. B 354, 155 (1995).

[45] J. Binnewies, B. A. Kniehl and G. Kramer, Z. Phys. C 65, 471 (1995).

[46] U. D'Alesio and F. Murgia, Phys. Rev. D 70, 074009 (2004). 